\title{
Realization of a Double-Barrier Resonant Tunneling Diode for Cavity Polaritons
}

\author{
H. S. Nguyen, ${ }^{1}$ D. Vishnevsky, ${ }^{2}$ C. Sturm, ${ }^{1}$ D. Tanese, ${ }^{1}$ D. Solnyshkov, ${ }^{2}$ E. Galopin, ${ }^{1}$ \\ A. Lemaître, ${ }^{1}$ I. Sagnes, ${ }^{1}$ A. Amo, ${ }^{1}$ G. Malpuech, ${ }^{2}$ and J. Bloch ${ }^{1, *}$ \\ ${ }^{1}$ Laboratoire de Photonique et de Nanostructures, LPN/CNRS, Route de Nozay, 91460 Marcoussis, France \\ ${ }^{2}$ Institut Pascal, PHOTON-N2, Clermont Université, Université Blaise Pascal, CNRS, \\ 24 avenue des Landais, 63177 Aubière Cedex, France
}

(Received 8 March 2013; published 3 June 2013)

\begin{abstract}
We report on the realization of a double-barrier resonant tunneling diode for cavity polaritons, by lateral patterning of a one-dimensional cavity. Sharp transmission resonances are demonstrated when sending a polariton flow onto the device. We show that a nonresonant beam can be used as an optical gate and can control the device transmission. Finally, we evidence distortion of the transmission profile when going to the high-density regime, signature of polariton-polariton interactions.
\end{abstract}

PACS numbers: 85.30.Mn, 42.65.Pc, 71.36.+c, 78.55.Cr

Resonant tunneling diodes (RTD) are primary elements of nanoelectronics providing negative differential resistance and other nonlinear properties $[1,2]$. They opened the way for many applications, such as high frequency oscillation [3], resonant tunneling transistor [4], or multiple-valued logic circuits [5]. Such double barrier structures, when brought to the quantum limit, reveal fascinating physics, such as the Coulomb blockade which was observed with electrons [6] and Cooper pairs [7]. Recently, resonant transmission in high quality factor photonic crystal cavities has allowed to evidence bistable behavior and implement optical memories, making use of the carrier-induced nonlinearity [8]. Transport of atomic Bose-Einstein condensates (BEC) through a double barrier structure has also been theoretically considered by several groups $[9,10]$, with interesting predictions related to nonlinear bosonic interactions. However, no experimental demonstration of these effects for BEC has been reported so far, due to the difficulty of creating an adequate potential profile for atomic BEC.

Cavity polaritons have appeared these last years as a very interesting system to investigate the physics of out of equilibrium condensates. They are exciton-photon mixed states arising from the strong coupling between the optical mode of a cavity and excitons confined in quantum wells [11]. Cavity polaritons propagate with a speed comparable to the speed of light ( $\sim 1 \%$ speed of light $)$ [12] thanks to their photonic component, and simultaneously show strong nonlinear interactions inherited from their excitonic component [13]. Cavity polaritons are now considered as a new platform for optical devices with many promising proposals for all-optical integrated logical circuits [14-17]. Experimental demonstrations of a polariton spin switch [18], polariton transistor [19,20], and of a polariton interferometer [21] have been recently reported. A key advantage of cavity polaritons is that the potential in which they evolve can be engineered at will, either by optical means [22-24], by depositing metallic layers on top of the cavity [25], by using a surface acoustic wave
[26], or by etching the cavity into lower dimensionality microstructures $[27,28]$.

In this Letter, we demonstrate the realization of a polariton RTD based on an innovative design of a wire cavity. Two micron-size constrictions are etched in the wire cavity and create two tunnel barriers, defining an isolated island, with discrete confined polariton states. Sending a polariton flow onto this double barrier structure, we observe resonant tunneling when the polariton energy coincides with the energy of one of the confined modes. We show that a nonresonant laser beam, focused onto the island, can modulate the RTD transmission with a peak-to-valley ratio as high as 28 . The device is, therefore, operating as an optical gate. Finally, we evidence strong asymmetry in the transmission profiles when going to the high-density regime. This is shown to be the signature of nonlinear polariton interactions within the island, as fully supported by numerical simulations.

The sample was grown by molecular beam epitaxy and consists in a $\lambda / 2$ microcavity with 28 (40) pairs of $\mathrm{Ga}_{0.8} \mathrm{Al}_{0.2} \mathrm{As} / \mathrm{Ga}_{0.05} \mathrm{Al}_{0.95} \mathrm{As} \lambda / 4$ layers in the top (bottom) distributed Bragg mirror. $12 \mathrm{GaAs}$ quantum wells $(7 \mathrm{~nm}$ thickness) are inserted in the structures. The quality factor of the microcavity amounts to 100000 and the Rabi splitting to $15 \mathrm{meV}$. Electron beam lithography and inductively coupled plasma dry etching were used to fabricate 1D microwires of $3 \mu \mathrm{m}$ width and $440 \mu \mathrm{m}$ length. The RTD is defined by a microstructure described in Figs. 1(a) and 1(b): two constrictions (width $=1.4 \mu \mathrm{m}$, length $=1 \mu \mathrm{m}$ ) surround an isolated island (width $=5.6 \mu \mathrm{m}$, length $=2 \mu \mathrm{m}$ ). As the 1D confinement potential is inversely proportional to the square of the wire width [29], the microstructure defines a double potential barrier and a OD polariton island [see Fig. 1(c)]. Microphotoluminescence experiments are performed at $10 \mathrm{~K}$ on single microwires using a cw monomode Ti:sapphire laser, focused onto a $2 \mu \mathrm{m}$ spot with a microscope objective $(\mathrm{NA}=0.55)$. A second cw monomode Ti:sapphire laser is used for experiments requiring 

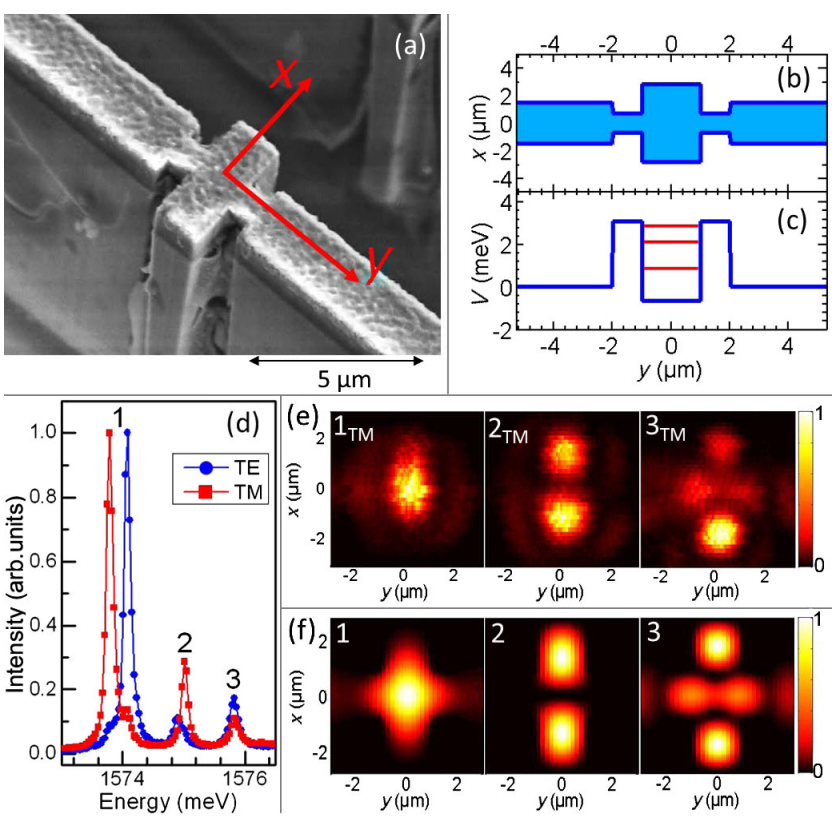

FIG. 1 (color online). (a) Scanning electron microscopy image of the polariton RTD. (b) Width profile of the polariton RTD. (c) Simulated potential along the wire in the region of the RTD (blue), and energy of confined states within the island (red). (d) Emission spectra measured on the island for TM (red circles) and TE (blue squares) detection polarization (excitation power $300 \mu \mathrm{W}$, laser energy $1.62 \mathrm{eV}$ ). (e) Spatially resolved emission of the three lowest energy confined polariton states for TM detection polarization. (f) Calculated emission pattern corresponding to (e).

simultaneously both resonant and nonresonant excitation. Polariton emission is imaged on a CCD camera coupled to a monochromator. The studied RTD corresponds to an exciton-photon detuning around $-10 \mathrm{meV}$ (defined as the difference between the energy of the photonic and excitonic modes).

We first characterize the polariton modes confined within the island, between the two tunnel barriers. Emission spectra of the island measured under TM (along $y$ axis) and TE (along $x$ axis) detection polarization are shown in Fig. 1(d). These spectra are obtained by exciting the island nonresonantly with excitation power well below condensation threshold. Three discrete polariton modes are observed for each polarization. Polarization splitting reflects the anisotropy of the confinement within the island. Spatial mapping of these confined modes is presented in Fig. 1(e) for TM polarization. Characteristic emission patterns with well defined emission lobes are observed in good agreement with simulations obtained solving a 2D Schrödinger equation for a particle of effective mass $m=6.1 \times 10^{-5} m_{\text {electron }}$ in a potential corresponding to that of the heterostructure. These measurements demonstrate that the two constrictions define an isolated island with well defined discrete states.

Let us now discuss polariton transport through this double-barrier structure. We first send onto the microstructure a polariton flow with a broad energy distribution. For this experiment, polaritons are injected with nonresonant excitation far from the microstructures (typically 50 to $80 \mu \mathrm{m}$ away). Figure 2(a) displays the spectral emission measured along the wire in TM detection polarization. The transmitted signal $(y>3 \mu \mathrm{m})$ presents a sharp threshold energy above which polaritons propagate across the island $\left(E>E_{b}=1575.8 \mathrm{meV}\right)$. This energy corresponds to the top of the tunnel barrier, and we deduce a barrier height of $V_{b} \approx 3.0 \mathrm{meV}$. Below $E_{b}$, the transmission is vanishing except for a few sharp resonances. The lowest one is observed at $1573.7 \mathrm{meV}$, and corresponds to the resonance of incident polaritons with the $1_{\mathrm{TM}}$ mode of the island. Transmission spectra for energy close to this resonance are reported in Fig. 2(b) for both detection polarizations: the observed peaks are split by the TE-TM splitting, showing that polariton polarization is preserved in the resonant tunneling. Notice that resonant tunneling transmission corresponding to $2_{\mathrm{TM}}$ (i.e., $1575.0 \mathrm{meV}$ ) is weak in Fig. 2(a). This feature can be explained considering the mode mismatch between the 1D incident polariton mode (symmetric along $x$ ) and the $2_{\mathrm{TM}}$ mode (antisymmetric along $x$ ). Finally, notice that the fringe pattern observed in the upstream side $(y<-3 \mu \mathrm{m})$ of Fig. 2(a) is due to the interference between incident polaritons and polaritons reflected on the double-barrier structure.

In the following, we describe how the transmitted intensity can be optically modulated, using a second laser beam focused on the island. To do this, we use the repulsive interaction of polaritons with a reservoir of excitons [23], which is locally injected in the island using a weak

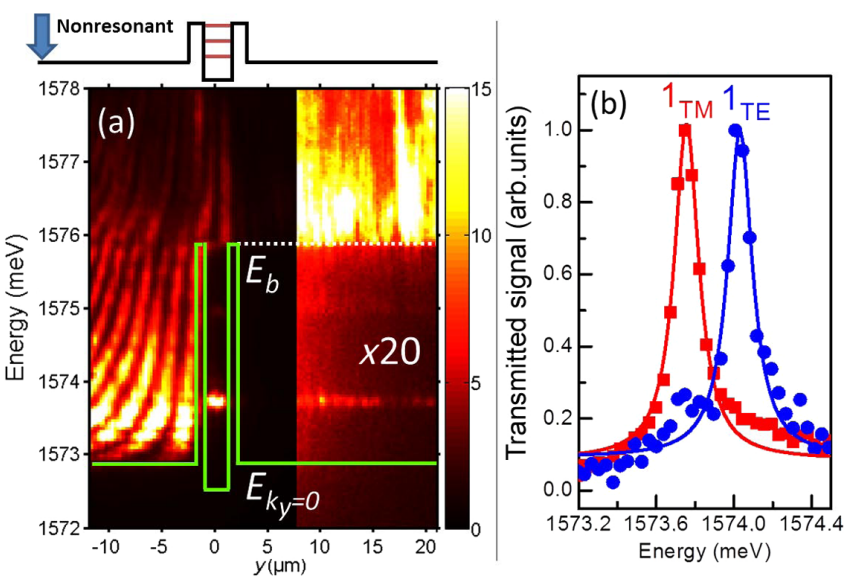

FIG. 2 (color online). (a) Spectrally and spatially resolved emission measured under TM detection polarization, when exciting the structure with a nonresonant laser beam of energy $1.62 \mathrm{eV}$ focused at position $y=-50 \mu \mathrm{m}$. (b) Transmission intensity measured under TM (red squares) and TE (blue circles) detection polarization. The signal is integrated between $y=$ $7 \mu \mathrm{m}$ to $y=15 \mu \mathrm{m}$. The red and blue lines are fits with two Lorentzians of $155 \mu \mathrm{eV}$ linewidth, limited by the detection resolution. 


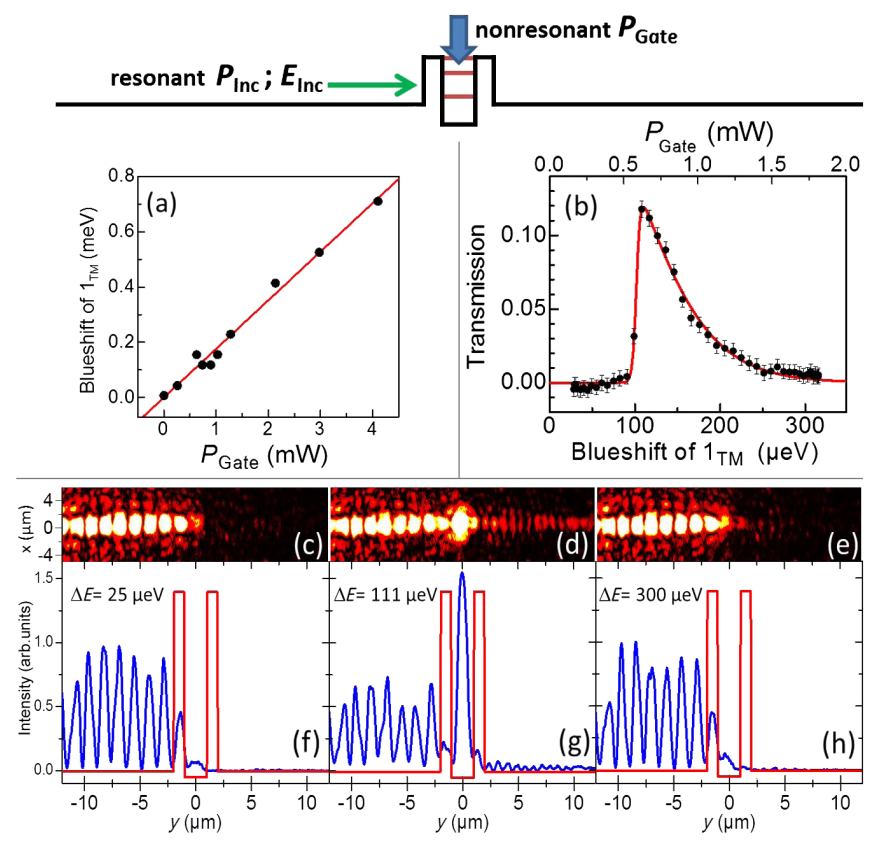

FIG. 3 (color online). (a) (black circles) Measured blueshift of the $1_{\mathrm{TM}}$ mode as function of the optical gate power $P_{\text {gate }}$. (red line) Linear fit with a $174 \mu \mathrm{eV} \mathrm{m} \mathrm{W}^{-1}$ slope. The energy of the laser gate beam is $1.62 \mathrm{eV}$. (b) Tunneling transmission as a function of the blueshift of the $1_{\mathrm{TM}}$ mode (bottom axis), or of $P_{\text {gate }}$ (top axis). The red line is a guide to the eye. The polariton flow is injected at $y=-80 \mu \mathrm{m}$ by a resonant laser of power $P_{\mathrm{inc}}=40 \mathrm{~mW}$ and of energy $E_{\mathrm{inc}}=1573.8 \mathrm{meV}$. (c)-(e) Spatially resolved emission measured for different values of $P_{\text {gate }}$ corresponding to a blueshift of the $1_{\mathrm{TM}}$ mode equal to (c) $25 \mu \mathrm{eV}$, (d) $111 \mu \mathrm{eV}$, and (e) $300 \mu \mathrm{eV}$. (f)-(h) Corresponding measured integrated intensity as a function of position (blue) and wire potential (red).

nonresonant optical excitation of power $P_{\text {gate }}$. When $P_{\text {gate }}$ is turned on, polariton-exciton interactions induce a $174 \mu \mathrm{eV} \mathrm{m} \mathrm{W}{ }^{-1}$ blueshift of the polariton modes confined within the island [see Fig. 3(a)]. We use this gate beam to control the transport of a monochromatic TM polarized polariton flow, which is sent onto the double-barrier structure, using a resonant laser beam (of energy $E_{\text {inc }}$ and power $P_{\text {inc }}$ ). We chose $E_{\text {inc }}=1573.8 \mathrm{meV}$, a value slightly larger than the energy of the $1_{\mathrm{TM}}$ mode (which lies at $1573.7 \mathrm{meV}$ ). Figure 3(b) reports the measured transmission for different values of $P_{\text {gate }}$. When the gate beam brings the energy of the $1_{\mathrm{TM}}$ mode into resonance with the polariton flow, a pronounced increase in the transmission is induced. This is further illustrated in Figs. 3(c)-3(h), where spatially resolved emission is monitored for values of $P_{\text {gate }}$ corresponding to energy of the $1_{\mathrm{TM}}$ mode below, at, and above the resonance. We clearly observed an enhanced luminescence signal in the downstream region at resonance [see Figs. 3(d) and 3(g)], which corresponds to an induced blueshift of $111 \mu \mathrm{eV}$. On the opposite side, a vanishing transmitted signal is observed for a blueshift of $25 \mu \mathrm{eV}$ or $300 \mu \mathrm{eV}$. These results prove that our device is indeed operated as an all optically controlled RTD, with a very high spectral selectivity. The peak (valley) transmission amounts to $11.1 \%(0.4 \%)$ resulting in a peak-to-valley signal ratio of 28 [30].

An interesting feature is revealed in the transmission spectrum presented in Fig. 3(b): the line shape is distorted with respect to a Lorentzian profile, with a more abrupt shape on the low power side. We show below that this is a direct evidence of polariton-polariton interactions [13] within the island, resulting in a nonlinear tunneling regime of the device. Let us first give a qualitative explanation of the observed asymmetric transmission profile. When $P_{\text {gate }}$ is slightly lower than the resonance power $P_{\text {res }}$, incident polaritons start to enter the island. As a consequence, the energy of $1_{\mathrm{TM}}$ undergoes an additional blueshift due to the interactions between polaritons confined in the island, which reduces the detuning between $E_{\text {inc }}$ and the energy of $1_{\mathrm{TM}}$. This mechanism thus provides a positive feedback that accelerates the passage to the resonant tunneling. On the contrary, when $P_{\text {gate }}$ slightly exceeds $P_{\text {res }}$, fewer polaritons enter the island. Thus, the additional blueshift decreases and the passage to off-resonant tunneling is slowed down due to a negative feedback.

Of course, this nonlinear regime occurs when the polariton density of the incident flow is large enough. If we reduce $P_{\text {inc }}$ sufficiently to enter the linear regime, then the luminescence from the island induced by $P_{\text {gate }}$ starts to dominate the emission spectra, and precise extraction of the transmission profiles becomes delicate. To unambiguously demonstrate the two regimes (linear and nonlinear), we performed experiments with a single laser beam (the one injecting the polariton flow) and probed the transmission profile when scanning the incident energy $E_{\text {inc }}$. For low incident power, a symmetrical transmission spectrum is measured characteristic of the linear regime [see Fig. 4(a) corresponding to $P_{\text {inc }}=5 \mathrm{~mW}$ ]. The transmission profile is well fitted by a Lorentzian of linewidth $\Gamma=27 \mu \mathrm{eV}$, attributed to the $1_{\mathrm{TM}}$ mode homogeneous linewidth. In analogy to the nondissipative case, where the tunneling transit time $\tau_{\text {transit }}[31]$ is twice the particle lifetime in the island [32], we can write here: $\Gamma=$ $2 \hbar / \tau_{\text {transit }}+\hbar / \tau_{\text {rad }}$, where $\tau_{\text {rad }}$ is the polariton radiative lifetime within the island, governed by the escape of the photon through the mirrors or the sidewalls of the structure. Moreover, the value of the transmission peak is given by $T_{\text {res }}=\tau_{\text {rad }} /\left(\tau_{\text {rad }}+\tau_{\text {transit }}\right) \approx 0.165$. Thus, we deduce $\tau_{\text {transit }}=172 \mathrm{ps}$ and $\tau_{\text {rad }}=34 \mathrm{ps}$. Notice that $\tau_{\text {rad }}$ is close to the nominal photon lifetime calculated from the quality factor of the nonetched structure, expected to be around 40 ps. For larger $P_{\text {inc }}$, asymmetry of the transmission spectrum develops as illustrated in Fig. 4(b) for $P_{\text {inc }}=$ $40 \mathrm{~mW}$. This asymmetrical profile, induced by polaritonpolariton interactions, is a mirror image of the one shown in Fig. 3(b), as here $E_{\text {inc }}$ is scanned with respect to the energy of the $1_{\mathrm{TM}}$ mode, whereas before the energy of $1_{\mathrm{TM}}$ 


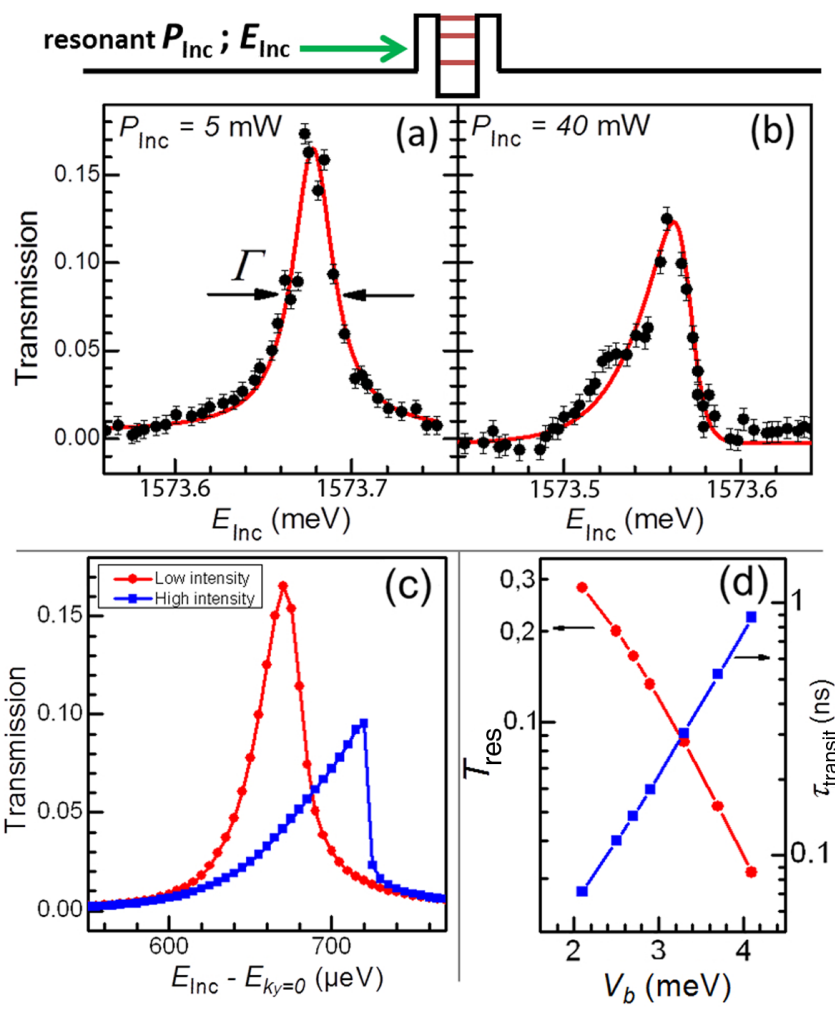

FIG. 4 (color online). (a), (b) Tunneling transmission measured as a function of $E_{\text {inc }}$ for (a) $P_{\text {inc }}=5 \mathrm{~mW}$ and (b) $P_{\text {inc }}=40 \mathrm{~mW}$. The red line in (a) is a Lorentzian fit of linewidth $27 \mu \mathrm{eV}$, while the red line in (b) is a guide to the eye. The polariton flow is injected at $y=-80 \mu \mathrm{m}$. (c) Simulation of the resonant tunneling transmission profile for low pump density (red circles) and high pump density (blue squares). Simulation parameters: polariton radiative lifetime $\tau_{\text {rad }}=34 \mathrm{ps}$, barrier potential $V_{b}=2.7 \mathrm{meV}$, interaction constant $g=0.4 \mu \mathrm{eV} \mu \mathrm{m}$, incident polariton density $65 \mu \mathrm{m}^{-1}\left(390 \mu \mathrm{m}^{-1}\right)$ for the low (high) pump density case. (d) Simulation of the resonant transmission $T_{\text {res }}$ (red circles) and the tunneling transit time $\tau_{\text {transit }}$ (blue squares) as a function of the barrier height.

was tuned with respect to $E_{\mathrm{inc}}$ using the gate beam. Such asymmetric shape of transmission profile due to nonlinear interaction has already been predicted for cold atom condensate transport through a double potential barrier [10], and has also been observed for resonant tunneling of guided light propagation through a photonic crystal microcavity [8]. To describe our experiments, we developed numerical simulations taking into account not only polariton-polariton interactions, but also the finite polariton lifetime, which is the specificity of our dissipative system. Figure 4(c) presents the numerical simulations of the transmission profile in the linear and nonlinear regime obtained with an interaction constant $g=0.4 \mu \mathrm{eV} \mu \mathrm{m}$ and a polariton density of 65 and $390 \mu \mathrm{m}^{-1}$. A 1D Gross-Pitaevskii equation has been used. Validity of this approximation has been confirmed by $2 \mathrm{D}$ simulations. The calculated profiles reproduce the observed features. However, while the simulation shown in Fig. 4(c) and the theoretical calculation for atom condensates [10] predict a blueshift of the resonant tunneling peak in the nonlinear regime with respect to the one in the linear regime, a redshift is observed in our experimental results [see Figs. 4(a) and 4(b)]. We attribute this discrepancy between theory and experiment to local heating of the sample in the highdensity regime.

Finally, we would like to discuss the performance of our polariton RTD device. In the present experiment, the peak transmission is limited to $16.5 \%$ because of the long tunneling time as compared to the photon lifetime. Further engineering of the tunnel barrier could allow controlling the tunneling transit time and thus optimizing the transmission. Indeed, Fig. 4(d) presents simulation of the resonant transmission and the tunneling transit time corresponding to different barrier heights. Our simulation points out that the resonant transmission coefficient can be significantly increased when reducing the barrier height. Concerning the dynamics of our RTD device, the optical gating dynamic is expected to be limited by the lifetime of the excitonic reservoir, which is around 400 ps [33]. Thus, we estimate that our device could operate at a frequency of several $\mathrm{GHz}$. We could envisage increasing this operating speed by using a resonant optical gate. Indeed, then the dynamics would be mainly governed by the polariton lifetime which is much shorter.

In conclusion, we have fabricated a polariton RTD exhibiting a resonant polariton transport through a double potential barrier structure. The device is gated by a lowpower nonresonant laser, which modulates the transmission within a peak-to-valley signal ratio of 28 . These results open the way for a new generation of integrated logical circuits by exploiting, for instance, the nonlinear transport of many RTDs in a more complex architecture [17]. Moreover, by reducing the size of the isolated island, the quantum regime could be reached: resonant tunneling transmission could become sensitive to singlepolariton nonlinearity [34]. In this regime, emission of nonclassical light is expected because of a polariton blockade, together with many fascinating features of Bose-Hubbard physics [35-38].

We thank Luc Le Gratiet for scanning electron microscopy. The work was supported financially by the 'Agence Nationale pour la Recherche' Projects "Perocai" and "Quandyde" (No. ANR-11-BS10-001), by the FP7 ITN "Clermont4" (No. 235114) and "Spin-Optronics" (No. 237252), by the french RENATECH network and the RTRA "Triangle de la Physique" Contract "Boseflow1D" and "2012-0397-Interpol."

*jacqueline.bloch@1pn.cnrs.fr

[1] T. C. L. G. Sollner, W. D. Goodhue, P.E. Tannenwald, C. D. Parker, and D. D. Peck, Appl. Phys. Lett. 43, 588 (1983). 
[2] H. Mizuta and T. Tanoue, The Physics and Applications of Resonant Tunneling Diodes (Cambridge University Press, New York, 1995).

[3] E. R. Brown, J. R. Söderström, C. D. Parker, L. J. Mahoney, K. M. Molvar, and T. C. McGill, Appl. Phys. Lett. 58, 2291 (1991).

[4] F. Capasso and R. Kiehl, J. Appl. Phys. 58, 1366 (1985).

[5] P. Mazumder, S. Kulkarni, M. Bhattacharya, J. P. Sun, and G. I. Haddad, Proc. IEEE 86, 664 (1998).

[6] C. Pasquier, U. Meirav, F. Williams, D. Glattli, Y. Jin, and B. Etienne, Phys. Rev. Lett. 70, 69 (1993).

[7] P. Joyez, P. Lafarge, A. Filipe, D. Esteve, and M. H. Devoret, Phys. Rev. Lett. 72, 2458 (1994).

[8] K. Nozaki, A. Shinya, S. Matsuo, Y. Suzaki, T. Segawa, T. Sato, Y. Kawaguchi, R. Takahashi, and M. Notomi, Nat. Photonics 6, 248 (2012).

[9] I. Carusotto and G. C. LaRocca, Phys. Rev. Lett. 84, 399 (2000).

[10] T. Paul, K. Richter, and P. Schlagheck, Phys. Rev. Lett. 94, 020404 (2005).

[11] C. Weisbuch, M. Nishioka, A. Ishikawa, and Y. Arakawa, Phys. Rev. Lett. 69, 3314 (1992).

[12] T. Freixanet, B. Sermage, A. Tiberj, and R. Planel, Phys. Rev. B 61, 7233 (2000).

[13] C. Ciuti, P. Schwendimann, and A. Quattropani, Phys. Rev. B 63, 041303(R) (2001).

[14] T. C. H. Liew, A. V. Kavokin, and I. A. Shelykh, Phys. Rev. Lett. 101, 016402 (2008).

[15] I. A. Shelykh, G. Pavlovic, D. D. Solnyshkov, and G. Malpuech, Phys. Rev. Lett. 102, 046407 (2009).

[16] I. A. Shelykh, R. Johne, D.D. Solnyshkov, and G. Malpuech, Phys. Rev. B 82, 153303 (2010).

[17] T. Espinosa-Ortega and T. C. H. Liew, arXiv:1302.1935.

[18] A. Amo, T.C.H. Liew, C. Adrados, R. Houdré, E. Giacobino, A. V. Kavokin, and A. Bramati, Nat. Photonics 4, 361 (2010).

[19] T. Gao, P.S. Eldridge, T. C. H. Liew, S. I. Tsintzos, G. Stavrinidis, G. Deligeorgis, Z. Hatzopoulos, and P. G. Savvidis, Phys. Rev. B 85, 235102 (2012).
[20] D. Ballarini et al., arXiv:1201.4071.

[21] C. Sturm et al., arXiv:1303.1649.

[22] E. Wertz et al., Nat. Phys. 6, 860 (2010).

[23] A. Amo, S. Pigeon, C. Adrados, R. Houdré, E. Giacobino, C. Ciuti, and A. Bramati, Phys. Rev. B 82, 081301 (2010).

[24] G. Tosi, G. Christmann, N. G. Berloff, P. Tsotsis, T. Gao, Z. Hatzopoulos, P. G. Savvidis, and J.J. Baumberg, Nat. Phys. 8, 190 (2012).

[25] C. W. Lai et al., Nature (London) 450, 529 (2007).

[26] E. A. Cerda-Méndez, D. N. Krizhanovskii, M. Wouters, R. Bradley, K. Biermann, K. Guda, R. Hey, P. V. Santos, D. Sarkar, and M. S. Skolnick, Phys. Rev. Lett. 105, 116402 (2010).

[27] J. Bloch, R. Planel, V. Thierry-Mieg, J. M. Gérard, D. Barrier, J. Y. Marzin, and E. Costard, Superlattices Microstruct. 22, 371 (1997).

[28] T. K. Paraïso et al., Phys. Rev. B 79, 045319 (2009).

[29] G. Dasbach, M. Schwab, M. Bayer, D. N. Krizhanovskii, and A. Forchel, Phys. Rev. B 66, 201201 (2002).

[30] See Supplemental Material at http://link.aps.org/ supplemental/10.1103/PhysRevLett.110.236601 for a movie showing the measured emission along the wire for different values of the optical gate power.

[31] M. Büttiker and R. Landauer, Phys. Rev. Lett. 49, 1739 (1982).

[32] U. Peskin, M. Galperin, and A. Nitzan, J. Phys. Chem. B 106, 8306 (2002).

[33] D. Bajoni, M. Perrin, P. Senellart, A. Lemaître, B. Sermage, and J. Bloch, Phys. Rev. B 73, 205344 (2006).

[34] A. Verger, C. Ciuti, and I. Carusotto, Phys. Rev. B 73, 193306 (2006).

[35] T. C. H. Liew and V. Savona, Phys. Rev. Lett. 104, 183601 (2010).

[36] M. Bamba, A. Imamoglu, I. Carusotto, and C. Ciuti, Phys. Rev. A 83, 021802 (2011).

[37] A.D. Greentree, C. Tahan, J.H. Cole, and L.C. L. Hollenberg, Nat. Phys. 2, 856 (2006).

[38] M. J. Hartmann, F. G. S. L. Brandão, and M. B. Plenio, Nat. Phys. 2, 849 (2006). 